\title{
A shadow fault detection method based on the standard error analysis of $I-V$ curves
}

\author{
M. BRESSAN ${ }^{1}$, Y. EL BASRI ${ }^{1}$, A. G. GALEANO ${ }^{3}$, C. ALONSO ${ }^{1,2}$ \\ ${ }_{1}$ CNRS; LAAS; 7 Avenue du Colonel Roche, F-31077, TOULOUSE, FRANCE. \\ 2 UNIVERSITE DE TOULOUSE; UPS, INSA; LAAS; F-31400, TOULOUSE, FRANCE. \\ ${ }_{3}$ Department of Electrical and Electronic Engineering, Universidad de los Andes, Bogotá, Colombia \\ E-mail: michael.bressan@laas.fr, corinne.alonso@laas.fr
}

Abstract: Shading on photovoltaic (PV) modules induces disproportionate impacts on power production. This paper presents a fault detection method able to identify anomalies on PV systems such as shading problems. The presence of localized shading on PV modules leads to an overheating of the shaded PV cells despite the activation of by-pass diodes. The temperature increase reduces considerably PV module performances and its lifetime. The presented method uses a simple equation, which corresponds to the normalized error (DE) of the comparison between the I-V curve in normal operation and the I-V curve in shading condition. The first derivative calculation gives the area of the detection in function of the PV voltage of the module (DE/DV). This defines whether one or several PV cells dissipate power. This phenomenon essentially occurs in the case of non-uniform irradiance received on PV modules and could impact PV modules performances. The detection method is explained in detail through the study of specific shadows simulations on PV modules. The results are validated through experimental tests on PV modules.

Keywords: Thermal dissipation, I-V curves analysis, shading problems, first derivative calculation, fault detection

\section{Introduction}

In recent years, photovoltaic (PV) modules have become a popular source of energy in both commercial and residential applications. This energy source has received much attention as an alternative form of electricity generation and is commonly present on buildings throughout the World.

Generally, PV modules are arranged in a way that maximizes the density of modules per unit of area rather than maximizing their efficiency. As a result, the incidence of shading over several modules is common in all applications, causing losses [1-2]. There are several causes of shading such as trees, adjacent buildings or soiling [3-4]. It is important to detect these failures because of partial shading can lead an annual 10-20\% reduction in power production or more in residential installations [5], due to both reduced irradiance on PV modules. Soiling may have a large effect on efficiency decreasing PV performances. Some studies [6-8] quantify performance decrease due to soiling. The study of Soteris [9] shows soiling affects seriously the performance of PV panels and it is necessary to clean immediately PV panels after dust episode. When a part of a PV module is shaded, some of its cells may work in reverse bias and reach breakdown voltage. The covered PV cells dissipate thermal power with the temperature increase. In extreme cases, this situation may lead to hot-spot problems, causing irreversible damage to the system. In order to protect shaded solar cells from breakdown voltage, commercial modules are equipped with by-pass diodes [10]. However, these by-pass diodes deform the I-V curves of the shaded modules when diodes are activated. 
Many authors have proposed solutions and algorithms to detect several failures on PV systems as hot-spot formation, soiling and aging degradation [11-13]. The electric method such as time domain reflectometry is able to detect failure position in PV cell string but it is not able to identify clearly if a shadow anomalies is present such as soiling. The study proposed by Hirata [13] consists in acquire I-V curves of PV modules string under low irradiance in order to detect parameter variation on series resistance, shunt resistance and diode factor. Based on this study, it exists a real interest to develop a method of fault detection able to prevent about shading problems and temperature increases despite the activation of the bypass diodes. It is important to investigate this problem because PV system are already confronted to shading problems in real operating.

It exists method able to detect online failures of PV systems and more particularly defects as shadows [14]. They all have a common point: the need of a proper shadow detection method. The first work [15] consisted of detecting the activation of bypass diodes. However, this is not enough to ensure a proper function of the PV cell. The presented method consists in detecting the activation of by-pass diode due to some shading scenario by calculating the first derivative (DI/DV) and the second derivative $\left(\mathrm{DI}^{2} / \mathrm{DV}^{2}\right)$. But this method does not permit to clearly identify if the shading is uniform or not on PV module. Moreover, it does not detect if a thermal dissipation problem of the covered PV cells exists despite the activation of the bypass diode.

In this paper, the presented shadow detection method consists in comparing I-V curves in normal operation issue to a validated solar model with I-V curves in shaded operation. The error issued from this comparison is normalized and derived in order to calculate a direct derivative in function of the PV module voltage. The main objective of this detection is to accurately identify anomalies such as shading problems on PV modules. The presence of localized shading on PV module causes a significantly temperature increase impacting PV performances and the PV module lifetime despite the activation of the by-pass diodes. This phenomenon is essentially characterized by a non-uniform shading. The difference of the I-V curves shape for each case of shading sets will permit to dissociate more easier an homogeneous shading of a non homogeneous and will facilitate the fault detection and the identification of the shadow anomaly. Moreover, this detection method should not perturb the PV production and it may operate with no need to be disconnected from its load thanks to an autonomous online I-V tracer [16]. For this study, and to validate the presented method, the I-V curves are traced using MP-160 I-V tracer (3kW). This paper is organized as follows, section 2 presents the necessity to develop a model considering the effect of shading on I-V curves. Moreover, some localized shading sets on PV module are performed to see the abnormal temperature increase of the shaded PV cell. The notion of "mismatch" on PV systems is explained. In function of these several thermal tests, the fault detection method is presented to prevent all localized shading problems on PV modules. Section 3 speaks about the experimental validation of the detection method on PV module. Several experimentally shading tests have been performed on two PV modules, one equipped with by-pass diodes and the other not. As a result, for the both cases, I-V curves traced and analyzed for each tests of shadows on PV modules. The last section concludes this work of detection.

\section{PV systems and shadows}

PV systems are composed of several elements, their power production is highly dependent on weather conditions and local environment. This section introduces these elements and their relationship with the external environme nt. 
When a PV cell is covered in a string, the overall current becomes limited by this covered cell increasing its temperature. An IR camera test is performed in order to see the PV module behavior in short circuit and in open circuit equipped of by-pass diodes in condition of localized shading. It is shown that despite the activation of the bypass diode and the presence of a non homogenous shading, it exists a thermal power dissipation of the covered PV cells. This theory review shows the necessity to have an accurate analysis and fault detection in order to avoid this phenomenon. The used PV module parameters are shown in Table 1.

Table 1: TE2200 PV module specifications

\begin{tabular}{cc}
\hline Variable & Value \\
\hline Cell type & Mono crystalline \\
Maximum power rating $\left(P_{\max }\right)$ & $250 \mathrm{Wp}$ \\
Open circuit voltage $\left(V_{o c}\right)$ & $37.5 \mathrm{~V}$ \\
Short-circuit current $\left(I_{s c}\right)$ & $8.8 \mathrm{~A}$ \\
Voltage at maximum power $\left(V_{m p}\right)$ & $30.05 \mathrm{~V}$ \\
Current at maximum power $\left(I_{m p}\right)$ & $8.4 \mathrm{~A}$ \\
By-pass diodes & 3
\end{tabular}

A string of PV modules in series is disconnected in order to perform shading tests on one PV module, in open circuit and in short-circuit. Fig.1 and Fig. 2 show four tests on PV modules established the December 10th 2015 during a sunny day. The ambient temperature recorded was $12^{\circ} \mathrm{C}$. The average wind speed was $2 \mathrm{~m} / \mathrm{s}$ and the global solar radiation at horizontal was $420 \mathrm{~W} / \mathrm{m}^{2}$. The several figures show the front of one PV module at vertical

(a)

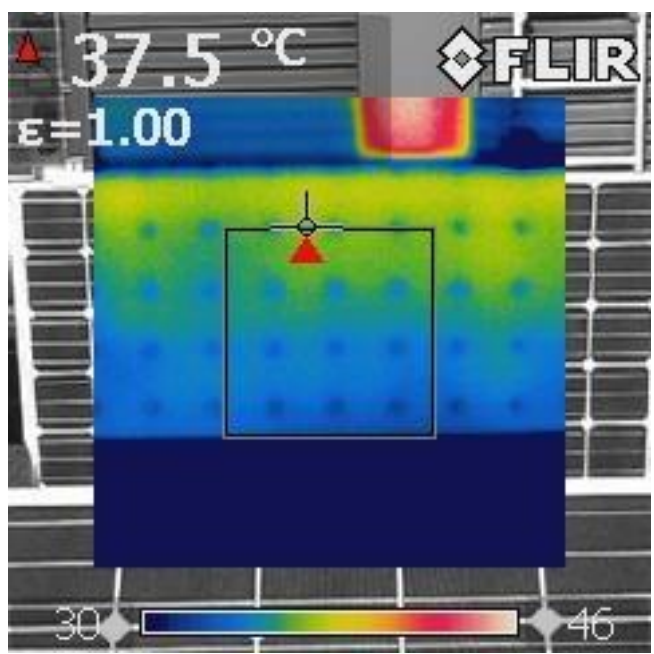

(b)

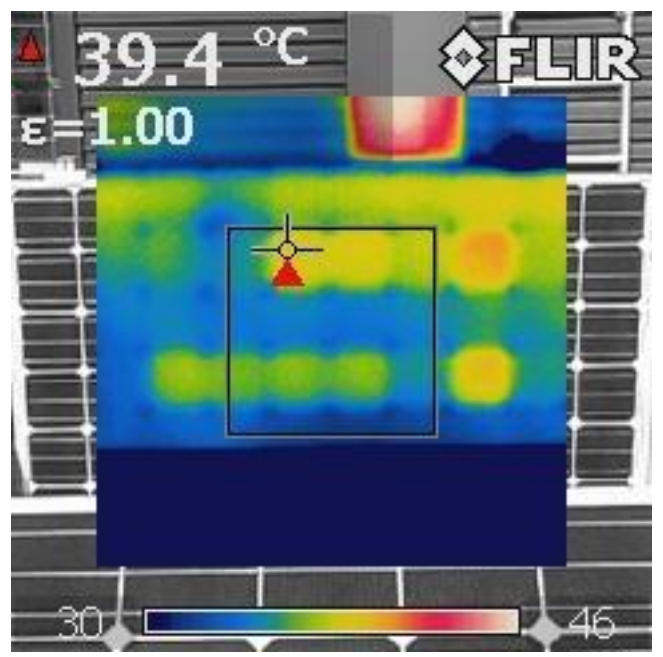

Fig. 1 : IR camera test on PV module in open circuit (a) and in short circuit (b) 
(a)

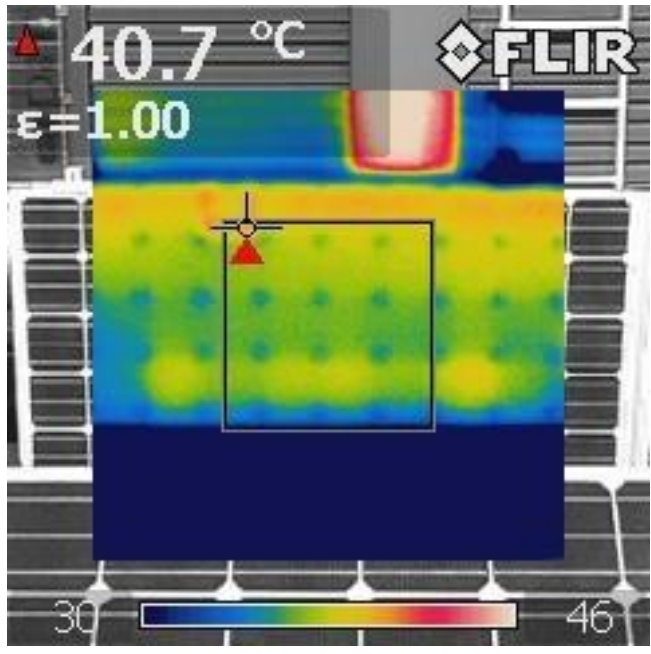

(b)

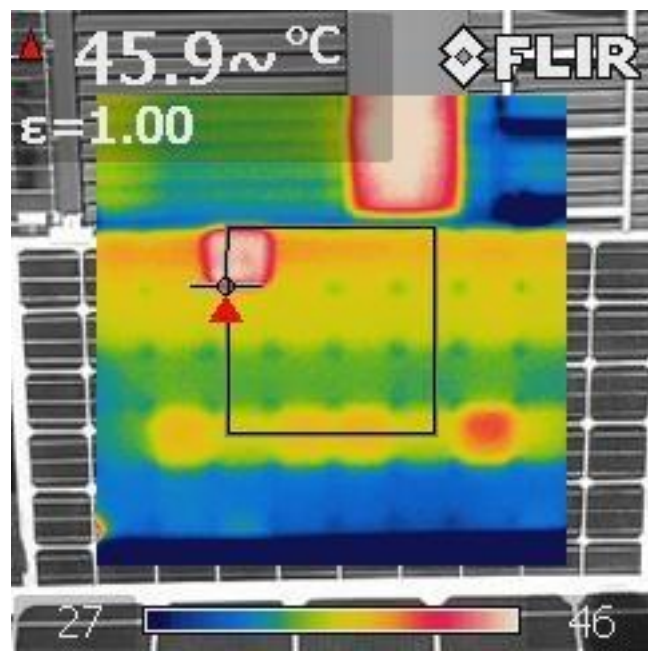

Fig. 2 : IR camera test on PV module with a covered PV cell in open circuit (a) and in short circuit (b)

Fig.1 (a) shows a homogenous temperature all over the PV module. After one hour, the initial temperature of the PV module in open circuit is about $33^{\circ} \mathrm{C}$. Temperature difference between the top and the bottom is due to the convective effects. Maximal temperature of the PV module in this case is $37.5^{\circ}$. If the PV module is in short circuit as shown in Fig. 1 (b), some PV cells starts to increase in temperature with a difference of $2^{\circ} \mathrm{C}$ with the first test. In the next test, just one PV cell of a sub-string is completely covered. First, the PV module is in open circuit, there is a homogenous temperature all over the PV module. The PV module is in short-circuit and after few minutes, its temperature increase contrary to the test in Fig.1 (a) despite the PV module is in open circuit. The convective effect has the same impact on the temperature increase visible in Fig. 2 (a). Then, the PV module is in short-circuit, a hot spot area at level of the covered PV cell is visible in red in Fig. 2(b). After one hour, a significantly increase of the temperature localized on the shaded PV cell is visible and it is evaluated at $45.9^{\circ}$. The shaded PV cell does not receive uniform illumination contrary to the others. The current in the PV module sub-string is limited by the shaded PV cell despite the activation of the by-pass diode of the sub-string. As a result, it operates under reversebias mode, dissipating power in the form of heat.

To resume, non-uniform shading has an important impact on PV performances despite the activation of by-pass diode. The IR thermography proved the presence of a hot-spot area of the shaded PV cell. It is necessary to understand the behavior of the PV module in shading conditions. A model is developed considering the reverse bias mode in shading conditions. This model will be used as reference in the presented fault detection method in function of input parameters such as solar irradiation and PV cells temperature. A fault detection method is necessary to prevent some losses related to shading problems. This work is particularly important for the diagnosis of faults and for improving the lifetime of PV systems. Analyzing I-V curves, it gives enough information in order to identify and to dissociate a uniform shading of a non-uniform shading. A fault detection method is developed around of the I-V curves analysis. This work will prevent immediately when a PV module is in shading conditions avoiding all form of dissipation. Moreover, it will allow to optimize efficiency of the PV module.

\subsection{Solar cell model}


The photovoltaic effect is the conversion of light into electricity. The classic model of a PV cell is governed by E quation (1) [17-18]. Equation (1) can be used to acquire I-V curve, which shows the behavior of the current and $t$ he voltage of a PV system.

$$
I=I_{p h}-I_{0}\left[\exp \left(\frac{\left(q\left(V+I \cdot R_{s}\right)\right)}{n k T}\right)-1\right]-\frac{V+I \cdot R_{s}}{R_{s h}}
$$

where $I_{p h}$ is the photocurrent (A), $I_{0}$ is the dark saturation current (A), $n$ is the ideality factor of the diode (1 to 2 ), $k$ is the Boltzmann constant $\left(1.3810^{-23} \mathrm{~J} /{ }^{\circ} \mathrm{K}\right), q$ is the magnitude of the electron charge $\left(1.60210^{-19} \mathrm{C}\right), T$ is the te mperature of the cell $\left({ }^{\circ} \mathrm{C}\right), R_{s}$ is the series resistance, $R_{s h}$ the shunt resistance.

However, most of the models in the literature do not take into account the effect of the reverse bias. A more precise model is proposed by Bishop [19] which incorporates the avalanche effect as a non-linear multiplication factor that affects the shunt resistance current term, shown below:

$$
I=I_{p h}-I_{0}\left[\exp \left(\frac{\left(q\left(V+I \cdot R_{s}\right)\right)}{n k T}\right)-1\right]-\frac{V+I \cdot R_{s}}{R_{s h}}-\underbrace{a \frac{V+I \cdot R_{s}}{R_{s h}}\left(1-\frac{V+R_{s} \cdot I}{V_{b r}}\right)^{-m}}_{\begin{array}{c}
\text { Multiplication factor on shunt } \\
\text { resistance }
\end{array}}
$$

where $a$ and $m$ are constant, $V_{b r}$ is the avalanche breakdown voltage (V).

By studying different PV cells in reverse bias, Bishop has noted a different evolution of the current. The avalanche breakdown in reverse bias is shown in red in Equation (2). Negative voltages for solar cells can occur at nonuniform illuminated PV generators, especially during partial shading of PV modules. The breakdown voltages for poly-Si cells are within the range $-12 \mathrm{~V}$ to $-20 \mathrm{~V}$, whereas for mono-Si cells the breakdown voltages can extend $12 \mathrm{~V}$ to $-30 \mathrm{~V}$ [20]. More precisely, when the PV module is partially shaded, the behavior of the I-V curve is different showing the reverse bias contrary to classic equation.

Fig. 3 shows I-V curves model for a generic PV cell with different illumination showing the avalanche effect of the PV cell in reverse bias. The breakdown voltage of the PV cell is estimated at $-15 \mathrm{~V}$.

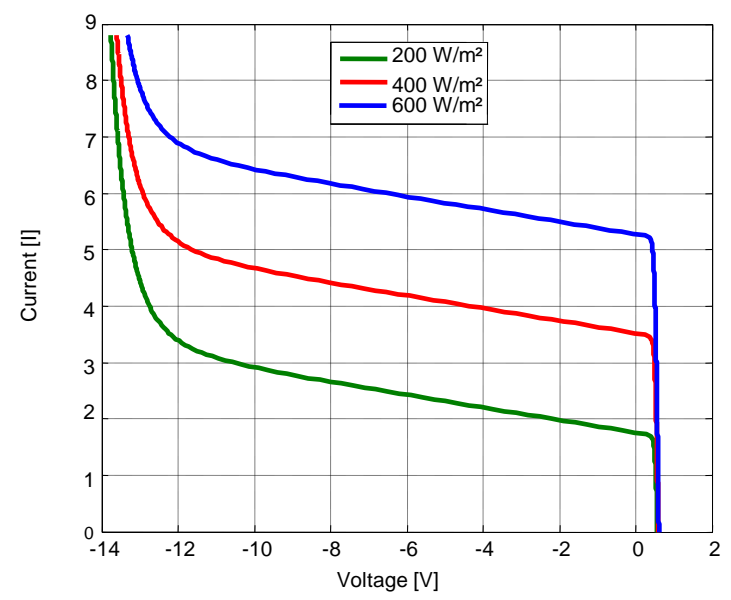

Fig. 3 : The I-V curves for different illuminations 
Several studies have analyzed these curves in detail such as [17] and [18]. In this work, a PV cell or plant is consi dered as partially shaded when a certain percentage of its surface is covered by a shadow. As a result, shaded cell $\mathrm{s}$ are forced to work in series with illuminated cells. Either each cell receives the same illuminated cells and oper ates at a lower current leading to a lower power production, or the short circuit current of the shaded cells is less $t$ han the string current so that it is operated ate the reverse characteristic, causing power to be dissipated and event ually the creation of hot-spots [21]. This phenomena causes permanent losses and reduces the reliability of the sy stem as shown with the thermography tests (Fig. 1 and in Fig.2).

\subsection{Partial shading and bypass diodes}

The solution used by the industry to protect the PV systems from partial shading is to equip them with bypass diodes. These diodes become biased when the voltage of the shaded PV cells becomes negative. This creates a short-circuit which limits the reverse voltage in the shaded cells [22].

Each cell group is protected by its own bypass diode, which has a protection threshold. This threshold limits the bias voltage of the shaded cells and therefore the dissipated power. When diodes detect a negative voltage, shaded cells are protected thanks to diodes activation. The threshold is estimated approximately at $-0.6 \mathrm{~V}$.

The model of I-V curve of this PV module is shown in Fig. 4 (a) for different percentage of shadows performed on one cell. If the string voltage exceeds the transmission voltage of the by-pass diode, it becomes active and shortcircuits the group of shaded PV cells. Theoretically, the breakdown voltage is not reached. In this simulation, there are one sub-string by-passed by the diode and two in normal operating. This leads to the apparition of two break points of the voltage as shown in Fig. 4 (b).

(a)

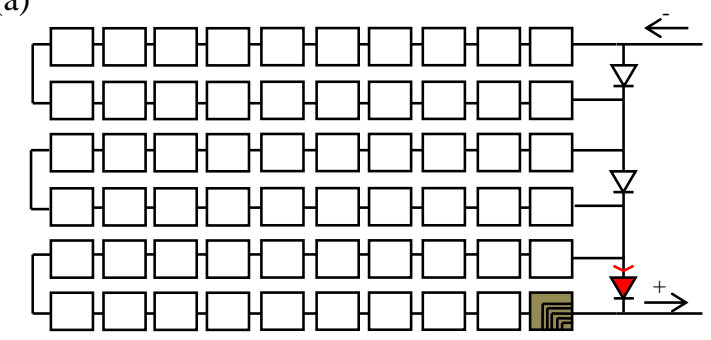

(b)

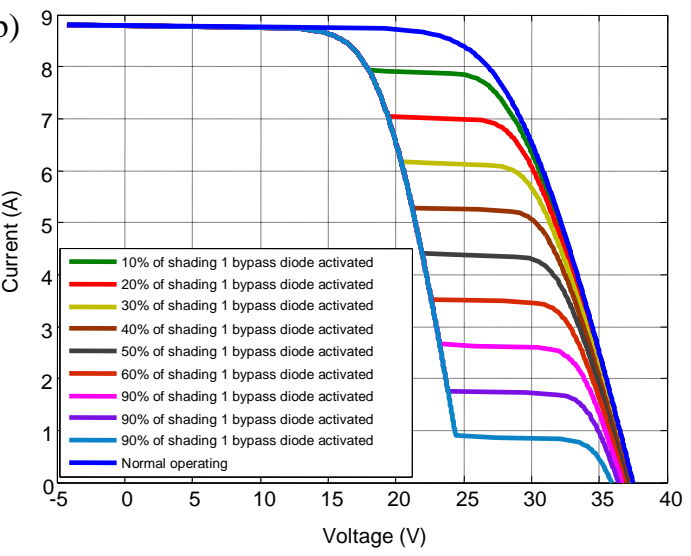

Fig. 4 : Shading tests simulation (a) and I-V curves results (b)

Most of the authors in the current literature works with shadows larger than a few cells [23-25]. Large shadows may represent the passage of clouds or the movement of trees, which may be a problem for medium to large PV plants. However, in smaller building integrated PV systems the PV plant may share the space with other objects leading to projected shadows which may cover only corners of the PV modules, i.e. shading only a few cells. Recurrent operation with small shadows is a subject generally overlooked by the current literature. In the previous part, localized and non-uniform shadows proved the presence of a hot-spot area with an increased temperature of the shaded PV cells. Despite the activation of the by-pass diode, it is shown with this temperature increase that the shaded PV cells dissipate power in the form of heat degrading PV performances and its lifetime. In the next part, 
a fault detection method is presented around I-V curves analysis. For this study, it is necessary to have an accurate solar model as reference. The used I-V model performances has been evaluated and has been considered satisfactory for the study [15].

\subsection{Method of detection of shading}

There is a vast collection of PV fault detection methods in the current literature. Those, which focus on the detection of shadows, can be roughly divided into power based or I-V based method. Power based detection methods focus their attention on the impact of partial shading in PV power production. The objective of these methods is to model the impact of the presence of the shadow over the PV plant depending on its geometric shape. This type of detection is mostly based on the precise and fast modeling of the characteristics of the PV modules [26]. This study proposes a simple mathematical model for the estimation of PV array power based on the consideration of shading geometry. The orientation of PV modules and location of the objects which will project shadows over the PV plant such as trees are important parameters taking into account for evaluating energy loss [27-28]. Most authors propose models which compare I-V curves from simulations with experimental data [2931]. However, these methods do not give enough information about PV modules behavior and performances in shading operating. The problematic of thermal power dissipation in shading condition is an important point to disseminate

The proposed method in this paper revisits the idea of using a first derivative calculation to detect the presence of a non-uniform shadows over a PV plant. Its main idea is that by monitoring the evolution of an I-V curve, it is possible to detect the activation of bypass diodes and to clearly identify and to dissociate a non-uniform shadow of a uniform shadow. Both don't have the same impact on PV performances and on its lifetime and this work prevent all thermal power dissipation of the shaded PV cell. The I-V curve acquired during shading conditions is compared to a reference I-V curve issue to a validated solar model. Their comparison is made through the estimation of the standard error using the current from the shaded and reference I-V curves, as defined in Equation (4).

$$
E(i)_{s t a n d a r d}=\frac{I(i)_{\text {reference }}-I(i)_{\text {shaded }}}{\max \left(I(i)_{\text {reference }}-I(i)_{\text {shaded }}\right)}
$$

Where $E(i)_{\text {standard }}$ is the standard error of the ith I-V curve point, $I(i)_{\text {reference }}$ is the current of I-V curve under reference conditions and $I(i)_{\text {shaded }}$ is the current of the I-V curve acquired under shaded conditions. The standard error is calculated for all of the points composing the I-V curve and its variation in relation to the PV voltage is used to monitor any changes in the shaded I-V curve as a whole. This derivative is calculated through the Equation (5).

$$
\frac{d E(i)_{s \tan d a r d}}{d V_{\bmod u l e}}=\frac{E(i+1)_{\text {stand dard }}-E(i)_{\text {stan dard }}}{V(i+1)_{\bmod u l e}-V(i)_{\bmod u l e}}
$$


To validate this method, the next section will apply it to a series of I-V curves with different shading scenarios. For each shading scenario, the evolution of the standard error will be analyzed in order to extract a general set of interpretation rules which can be used to clearly identify some shading problems increasing the shaded PV cells temperature. The experimental study shows the area of detection in order to dissociate the uniform-shadows of the non-uniform shadows despite the activation of the by-pass diode.

\section{Experimental study of the detection method}

The experimental shading scenarios used in this section have the objective of providing a set of realistic conditions to study the effectiveness of the new proposed detection method. These several I-V curves analysis confirm that the shaded PV cells which is non-uniform, dissipates power in the form of heat as explained in Section 2. The experimental setup will be presented first, giving the several shading scenarios chosen for the study. These are followed by the analysis of the results for each shadow scenario.

\subsection{The experimental setup}

The PV module used during these experimental tests is still the TE2200. The I-V curves are traced using the MP$160 \mathrm{I}-\mathrm{V}$ tracer, which provides 265 measurement points between open-circuit voltage to short-circuit current. A total of 6 sets of experimental tests were conducted to study this new detection method. Each set is composed of a certain shading area, a shadow size and the state of the bypass diodes.

The shading set 1 was chosen to clearly study how the detection method reacts to the presence of the non-uniform shadow on PV module without by-pass diodes. The shading set 2 is studied in comparison with the shading set 1 to study the difference between the activation of the bypass diodes and the presence of a localized shaded PV cell without by-pass diode. The shading set 3 studies how the detection method can identify a homogeneous shadow with the activation of one by-pass diode. Thus, the shading size used is 20 cells all of which have $50 \%$ of their area shaded. The shading set 4 and 5 study the activation of 2 bypass diodes. The shadow used in these cases covers two cells on the interface between two PV cell groups protected each by a different bypass diode. The shading area of the set 4 is chosen to be $50 \%$ to simulate a shading scenario while the shading area of $100 \%$ in set 5 represents another case of non homogenous shading. Finally, the shading set 6 studies the detection of a homogeneous shadow which activates two bypass diodes. It shares the same characteristics of the shading set 3 . An overview of the shading scenarios used in this study is given in Table 2.

Table 2 : Scenarios of shading sets performed over PV module equipped or not of bypass diodes

\begin{tabular}{|c|c|c|c|}
\hline Shading Set & Shading Area & Shadow Size & Bypass Diodes \\
\hline 1 & Variable $(10 \%-100 \%)$ & 1 Cell & Removed \\
\hline 2 & Variable $(10 \%-100 \%)$ & 1 Cell & 1 Active \\
\hline 3 & Fixed $(50 \%)$ & 20 Cells & 1 Active \\
\hline 4 & Fixed $(50 \%)$ & 2 Cells & 2 Active \\
\hline 5 & Fixed (100\%) & 2 Cells & 2 Active \\
\hline 6 & Fixed (50\%) & 40 Cells & 2 Active \\
\hline
\end{tabular}

3.2 Shading set 1 - Overall study of the presence of the reverse bias on I-V curves of the PV shaded cell 
The results for the shading set 1 are given in Fig 5. The shadow scenario is shown in Fig. 5 (a), which represents the variation of the shading area. The presence of the reverse bias characterized by the avalanche effect and caused by each shadow is visible on I-V curves in Fig. 5 (b). The results of the detection method are shown in Fig. 5 (c).

(a)

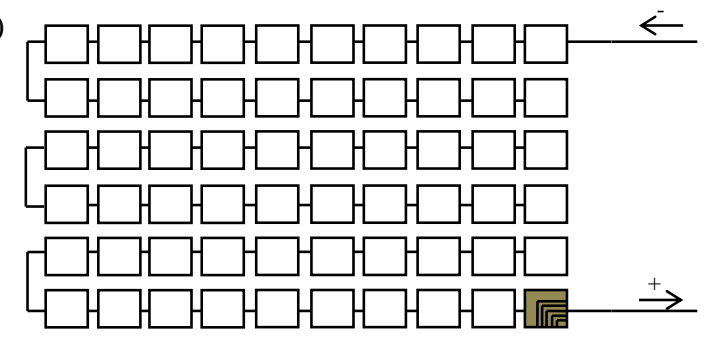

(b)

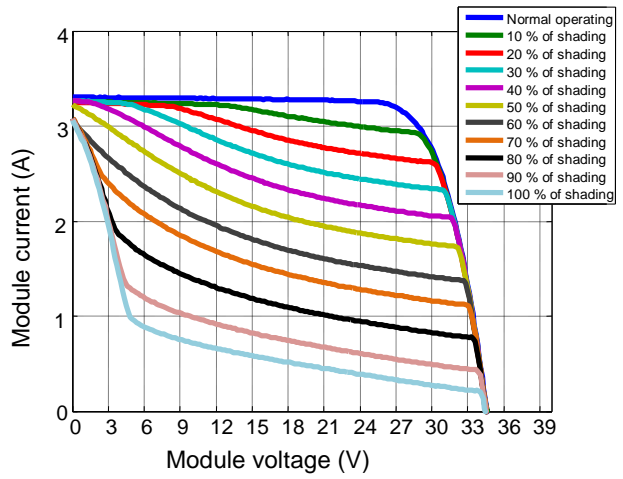

(c)

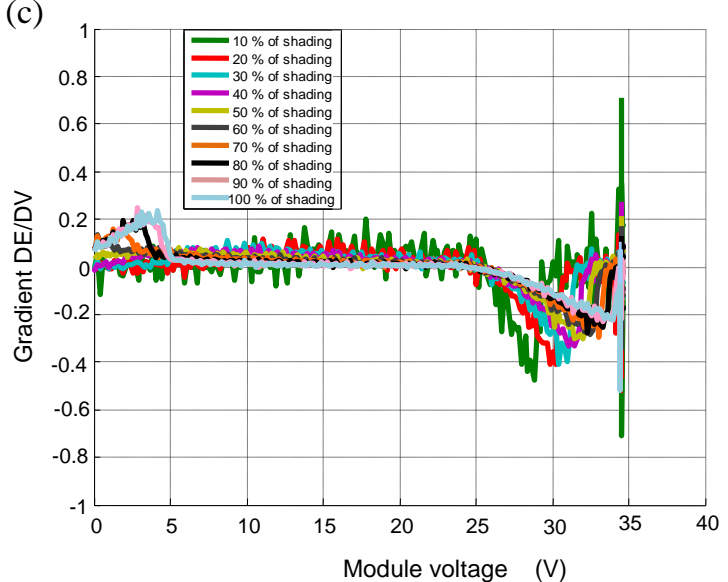

Fig. 5 : Results for the shading set 1: (a) the shadow scenario, (b) the I-V curves for each shadow scenario and (c) the results of the detection method.

In Fig. 5 (b), the avalanche breakdown of the shaded PV cell is more visible on I-V curves with an high percentage of shading on PV cell. The I-V curve slope for $100 \%$ of shading strongly changes contrary to $10 \%$ of shading As a result, this slope characterizes this avalanche effect of the shaded PV cells which dissipate power in the form of heat damaging PV module on the long term and reducing its lifetime. The results from the detection method in Fig. 5 (c) shows two areas of interest, the first from $0 \mathrm{~V}$ to $5 \mathrm{~V}$ and the second from $25 \mathrm{~V}$ to $35 \mathrm{~V}$. The first area $(0$ $5 \mathrm{~V}$ ) has a positive peak that rises with the shading area. This peak is clearly caused by the presence of the avalanche effect and represents a growing error in comparison with the reference. A growing error at such low voltages could be interpreted as a shadow that has an overall impact on the power production, in this case due to the absence of the bypass diodes. The second area of interest in Fig. 5 (c) $(25 \mathrm{~V}-35 \mathrm{~V})$ shows a negative peak that becomes lower with the shading area. This peak shows that the error is becoming lower as the voltage is close to the open-circuit voltage. In normal operating conditions, the bypass diodes will be connected and operational.

\subsection{Shading set 2 - Bypass diodes and non homogenous shadows}

In the shading set 2, the bypass diodes are connected. The shadow and active bypass diode are shown in Fig. 6 (a). The effect of the shadow over the I-V curve is shown in Fig. 6 (b) while the results of the detection method are shown in Fig. 6 (c). 
(a)

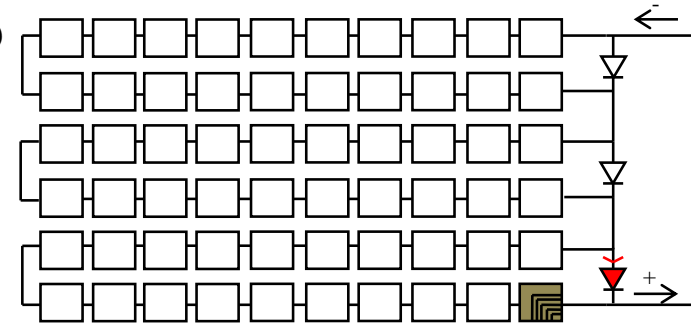

(b)

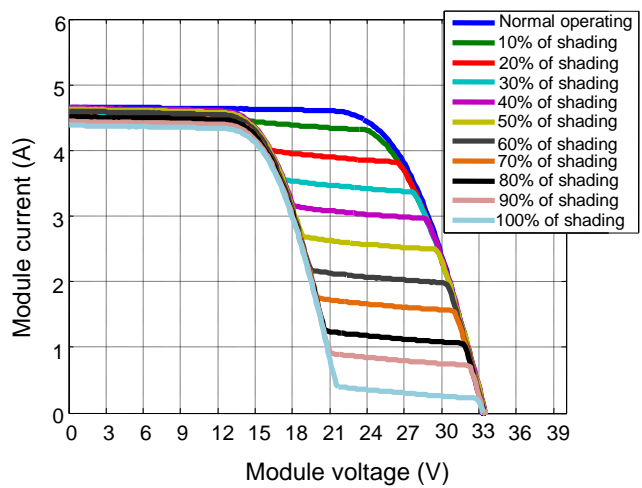

(c)

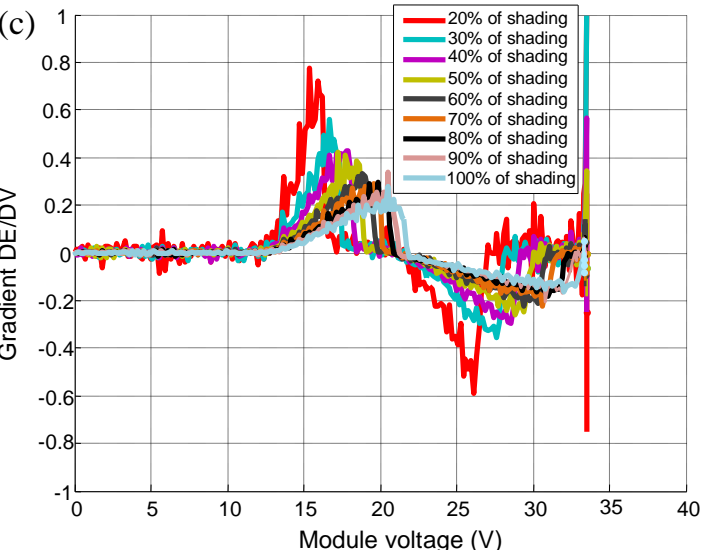

Fig. 6 : Shading set 2 operation over PV modules (a), I-V curves results (b) and first derivative results (c)

By comparing Fig. 5 (b) and Fig. 6 (b), it is possible to see that the presence of the bypass diode introduces a step.

The slight slope present in Fig. 6 (b) between $15 \mathrm{~V}$ to $33 \mathrm{~V}$ for the different shading scenario shows that the shaded cell is a non homogenous shading. Moreover, this slope characterizes a part of the avalanche breakdown of the PV shaded cell despite the activation of the by-pass diode. In the point of view of I-V curves, this means the shaded PV cell dissipates power in the form of heat despite the activation of the by-pass diode. It is the detection of this kind of operating condition, the main objective of the new proposed method. The results shown in Fig. 6 (c) also have two areas of interest, the first from $14 \mathrm{~V}$ to $21 \mathrm{~V}$ and the second from $21 \mathrm{~V}$ to $33 \mathrm{~V}$. The first area of interest $(14 \mathrm{~V}-21 \mathrm{~V})$ shows a positive peak whose value decreases with the shading area. An abrupt positive change in the variation of the error is linked to the presence of the shadow, as described in the shading set 1 . However, in this shading set the positive peak is located at a higher voltage giving a clear indication of the correct operation of the bypass diode. By comparing the first area of interest in Fig. 5 (c) and Fig. 6 (c), it is clear that the correct operation of the bypass diode can be directly linked to the voltage in which the positive peak appears. The second area of interest $(21 \mathrm{~V}-33 \mathrm{~V})$ shows a negative peak whose value decreases with the shading area. This behavior is similar to the one observed for the second area of interest in Fig. 5 (c) and validates the impact of a non homogenous shadows despite the activation of the bypass diode. The cross comparison of the results from the shading sets 1 and 2 shows that positive peaks are linked to the bypass diodes activation with the presence of the avalanche effect characterizing a non homogenous shading. To validate this idea, the shading set 3 will study the presence of these peaks with this time an uniform shading test.

\subsection{Shading set 3 - Bypass diodes and homogenous shadows}

The shading set 3 shows how the detection method reacts to the activation of the bypass diode with an homogenous shadow. The shadow shape and active bypass diode are shown in Fig. 7 (a). The effect of the shadow over the I-V curve is shown in Fig. 7 (b) while the result of the detection method is shown in Fig. 7 (c). 
(a)

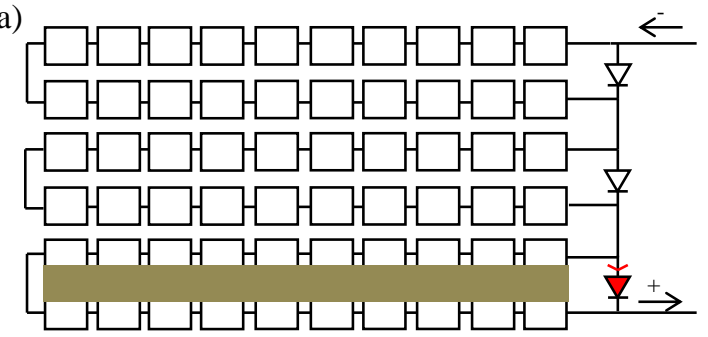

(b)

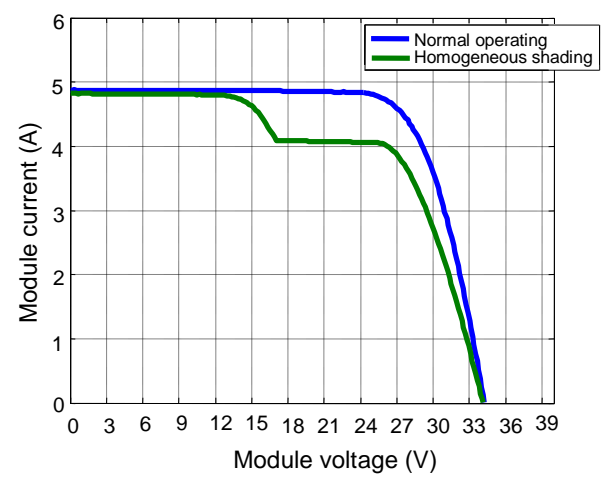

(c)

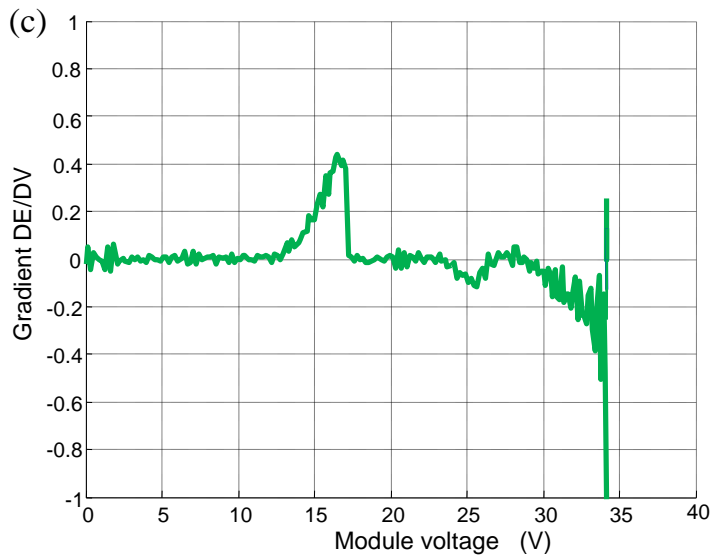

Fig. 7 : Shading set 3 - (a) the shadow shape, (b) the impact of the shadow over the I-V curve and (c) the result from the detection method

Clearly, the slope of the region situated between $15 \mathrm{~V}$ and $27 \mathrm{~V}$ is less pronounced than the slope visible in Fig 6 (b). This means that it is possible to distinguish an uniform shadows of a non uniform shadow. Moreover, the presence of this pronounced slope shown in Fig. 6 (b) is due to the avalanche effect of the shaded PV cell. By supposition, this shaded PV cell could dissipate a part of the DC power of others cells despite the activation of the by-pass diode. As a result, it is essential to avoid this phenomena and to prevent against non homogenous shading. The result in Fig. 7 (c) shows a clear positive peak from $12 \mathrm{~V}$ to $17 \mathrm{~V}$ and two noisy negative peaks around $25 \mathrm{~V}$ and $33 \mathrm{~V}$. In this case, the shadow can be considered as homogeneous over the whole PV cell group, which means that the avalanche effect can be almost neglected. In the absence of this avalanche effect, the positive peak prevails over the two small negative peaks showing that the protection provided by the bypass diode can be considered as enough. This result validates the link between the positive peaks and the bypass diodes as well as the link between the negative peaks and the avalanche effect of the shaded PV cells. Now, three others cases where the number of bypass diodes is different will be used to study how this new method can be used to detect the number of active bypass diodes and their effectiveness.

\subsection{Shading sets 4 and 5 - Several bypass diodes and non homogenous shadows}

The shading set 4 and 5 seek to study how the activation of several bypass diodes can be detected by the proposed method and if the non homogenous shading effect can still be distinguished under these shading conditions. Thus, the shadow shape in these cases is located in the interface between two PV cell groups, forcing the activation of two bypass diodes. The shadow shape and active bypass diode are shown in Figs. 8 (a) and 8 (b). The effect of the shadow over the I-V curve is shown in Figs. 8 (c) and 8 (d) and the results of the detection method are shown in Fig. 8 (e). 
(a)

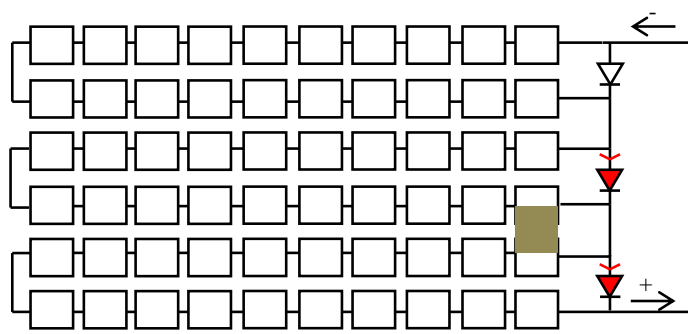

(c)

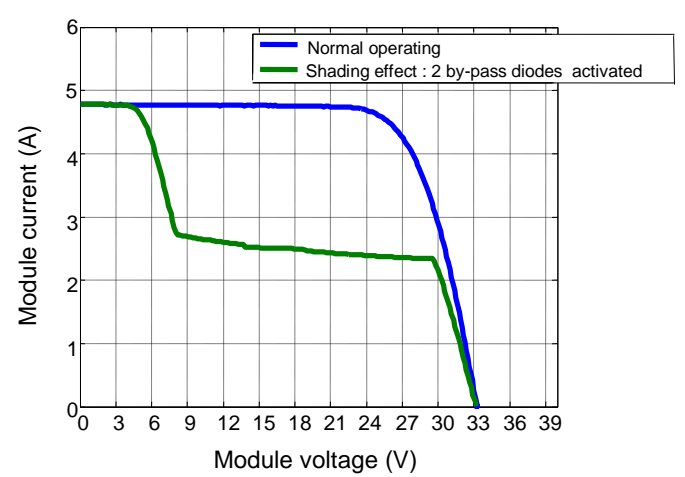

(b)

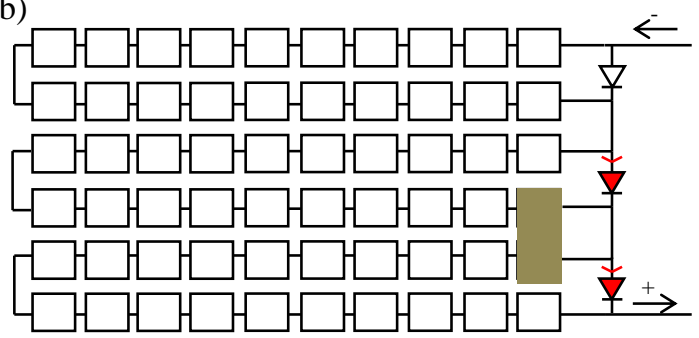

(d)

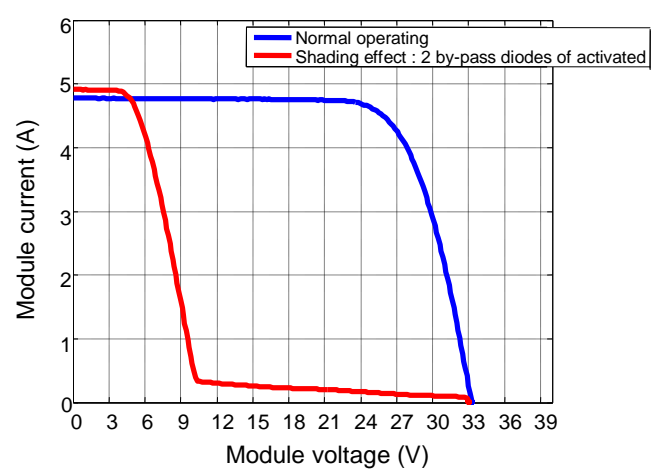

(e)

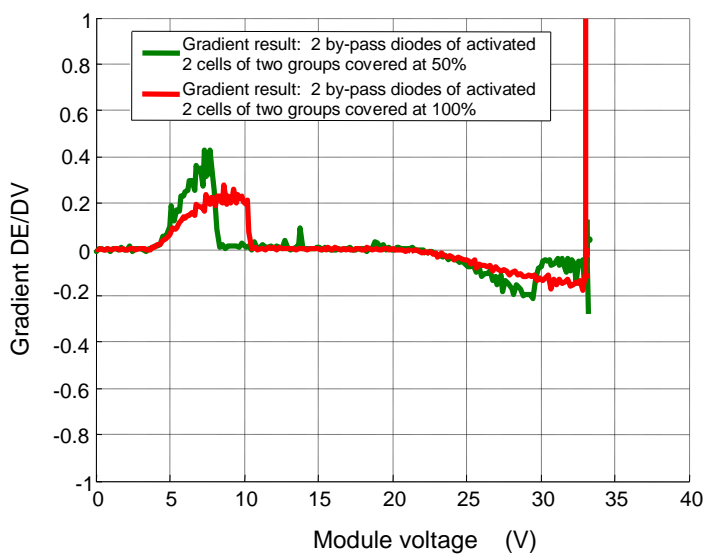

Fig. 8 : Shading sets 4 and 5 - (a) and (b) the shadow shape, (c) and (d) the impact of the shadow over the I-V curves, (e) the results from the detection method.

The results shown in Fig. 8 (e) validate the two areas of interest observed in Figs. 5, 6 and 7.

The first area of interest is located from $5 \mathrm{~V}$ to $10 \mathrm{~V}$ and has two positive peaks whose value decreases with the shading area. These results confirm the link between positive peaks and the activation of bypass diodes. The occurrence of these peaks at a lower voltage also indicates that a higher number of bypass diodes is active and, by consequence, that the shadow is covering a larger part of the PV module.

The second area of interest is located from $21 \mathrm{~V}$ to $33 \mathrm{~V}$ with the two negative peaks representing the presence of the non uniform shading visible by the slope more pronounced ( $9 \mathrm{~V}$ to $30 \mathrm{~V}$ ) in Fig 8 (d) and (e). This result also confirms the link between negative peaks and the avalanche effect of the shaded PV cells due to a non homogenous shading. The appearance of these negative peaks at similar voltages than those in Figs. 6 and 7 indicates that the same principle can be used to detect the presence of a shading no matter the size of the shadow such as soiling.

3.6 Shading set 6 - Large homogeneous shading 
The shading set 6 has the objective of confirming the detection of the activation of bypass diodes for larger shadows without pronounced slope visible in Fig. 8 (c) between $9 \mathrm{~V}$ and 30V. The shadow is shown in Fig. 9 (a), its effect in the I-V curve is shown in Fig. 9 (b) and the results from the detection method are shown in Fig. 9 (c).

(a)

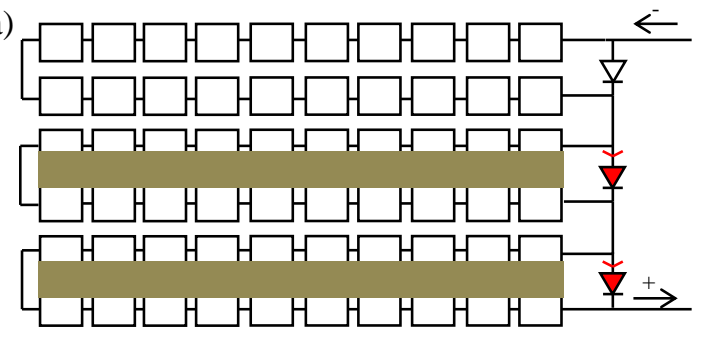

(b)

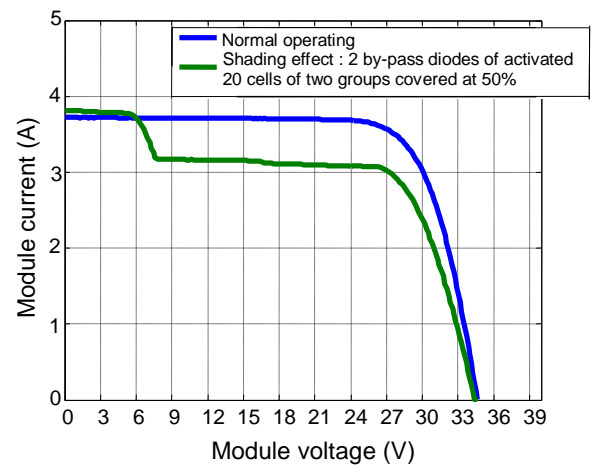

(c)

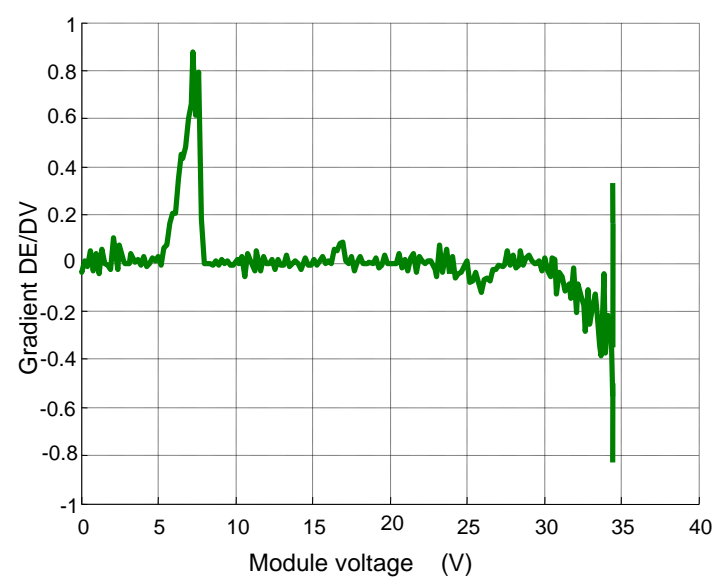

Fig. 9 : Shading set 6 - (a) the shadow scenario, (b) the impact of the shadow over the I-V curve and (c) the results from the proposed detection method.

The result from Fig. 9 (c) confirms that a positive peak is directly linked to the activation of bypass diodes. When a positive peak appears at voltages much lower than the open-circuit voltage, it means that the shadow is large and several bypass diodes are active. Since the negative peak is very close to the open-circuit voltage, it can be interpreted that the shadow is homogeneous without a pronounced slope in the region between $8 \mathrm{~V}$ and $27 \mathrm{~V}$.

\subsection{Summary of the experimental study}

To summarize the results from the experimental study, Fig. 10 shows the results from the detection method for several shading cases. 


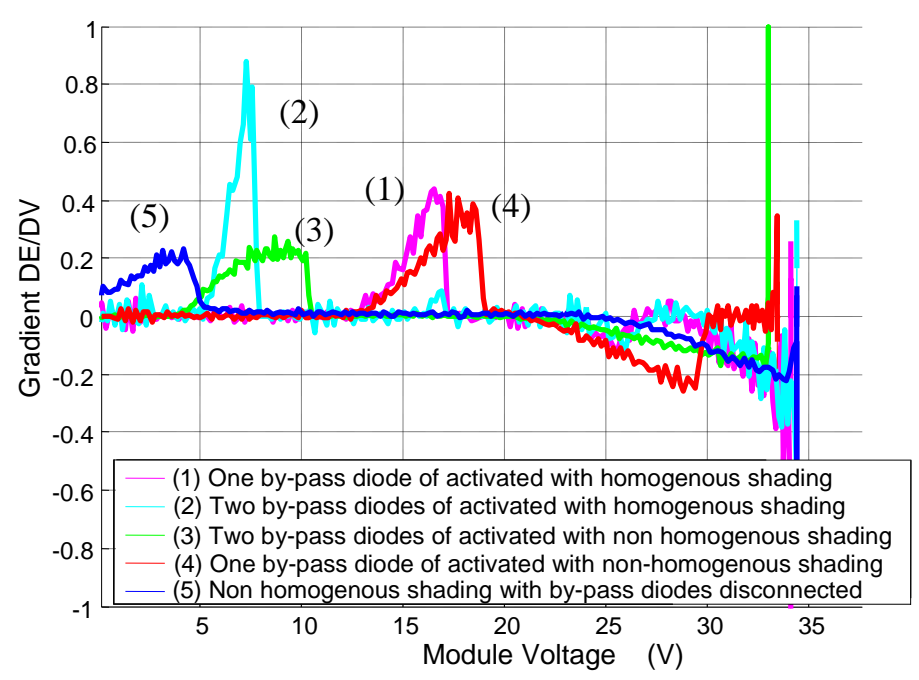
PV module voltage for each tests of shading on PV module

The results validate the idea that positive peaks are associated with the activation of bypass diodes and negative peaks are associated with the presence of the avalanche breakdown of the shaded PV cells on I-V curves due to a non homogenous shading.

The positive peak shifts to lower voltage values as the shadow area becomes wider and more bypass diodes become active, as can be seen in cases 1 through 4 in Fig. 10. In case 5, where no bypass diode is activated, there is a positive peak close to the short circuit current, further corroborating the avalanche breakdown in reverse bias of the shaded PV shaded cells. The negative peak shifts to voltage values close to the open-circuit voltage for severe cases of non-uniform shading. Since all the negative peaks have a similar point of origin (around $20 \mathrm{~V}$ ) it is the distance between these two points that can be used to determine the severity of the presence of this avalanche effect and more particularly the presence of a non-homogenous shading. For each shading set, different areas are visible and may be dissociated in order to perform the fault detection. This fault detection method could be implemented in a microcontroller and in embedded system. The position of the maximum amplitude in each case of shading set is different whether in the positive side than the negative side in function of the PV module voltage An important remark is that the noise can be an issue when evaluating the presence of the peaks and their occurrence. To avoid false detections, it is recommended to establish an uncertainty zone around zero $\mathrm{dE} / \mathrm{dV}$ and around $\mathrm{V}_{\mathrm{OC}}$. Peaks within these areas do not bring useful information to the detection and should be ignored. To summarize this part, for each shading set, an area on the calculation of DE/DV have been identified in order to detect and to dissociate a non-uniform shading of an uniform shading despite the activation of the bypass diode. The presence of the avalanche breakdown on I-V curves when the by-pass diode is activated and the non homogenous shading could have an impact on aging PV modules. Indeed, this pronounced slope traduces the fact that the shaded PV cells could dissipate power and could have important impact on the PV modules lifetime and on their effectiveness.

\section{Conclusion}

The shading effect on PV module is one of the main causes of inefficiency and degradation of PV modules losses. It is difficult to quantify precisely the resulting losses and the impact on PV module aging, especially in urban 
areas. Despite the use of by-pass diodes to protect PV modules, the presence of the avalanche effect of the shaded PV cell is visible on experimental I-V curves test with non-homogeneous shading. Some localized shaded PV cell dissipate power in the form of heat as shown in Section 2. It is shown in this study the possibility to detect and to disseminate shading forms which have not the same impact on the long term on PV modules performances for example for the case of the presence of soiling. As a result, this phenomenon could impact the PV module performance and could probably accelerate its premature aging.

This study has shown that it is possible to detect and identify the avalanche effect of the shaded PV cell through the presented analysis of I-V curves. Moreover, it is possible to dissociate a homogenous shading of a non homogenous shading. Several shading sets were performed on a PV module to validate the detection method proposed in this work. This method is based on the comparison of I-V curves obtained in normal operating conditions with those obtained under shading conditions. The first derivative calculation between the standard error and the PV module voltage can detect the activation of bypass diodes or other effects such as the dissipation power due to non homogenous shading on PV modules.

\section{Acknowledgements}

It exists a real interest to deepen this work. It will be interested to implement this method of fault detection in an embedded system. To avoid the use of solar model and additional sensors, the I-V curve reference may be the lasted data stored in memory to compare with the one in real time. The first perspective is to investigate the development of an I-V curve tracer in real time and in low power without perturb the PV production. This would permit to validate this analysis in real condition. The complexity to develop it for a PV chain is a technological research subject at investigate. The second perspective is to verify the relevance of this fault detection method through PV modules connected in series analysis.

\section{References}

[1] M.C Alonso-Garcia, J.M Ruiz, F. Chen lo, "Experimental study of mismatch and shading effects in the I-V characteristic of a photovoltaic module", Solar Energy Materials \& Solar Cells 90, 2006, 329-340

[2] A. Woyte, J. Nijs, R. Belmans, "Partial shadowing of photovoltaic arrays with different system configurations: literature review and field test results”, Solar Energy, Volume 74, Issue 3, March 2003, Pages 217-233

[3] Y. Sun, X. Li, R. Hong, H. Shen, "Analysis on the Effect of Shading on the Characteristics of Large-scale ongrid PV System in China", Energy and Power Engineering, 2013, 5, 215-218

[4] E. Lorenzo, R. Moreton, I. Luque,'Dust effects on PV array performance: in-field observations with nonuniform patterns", PROGRESS IN PHOTOVOLTAICS: RESEARCH AND APPLICATIONS, Prog. Photovolt: Res. Appl. 2014; 22:666-670, online library.

[5] C. Deline, B. Marion, "A performance and Economic analysis of distributed Power Electronics In Photovoltaic systems", NREL, prepared under Task No. PVD9.1410.

[6] F. Mejia, J. Kleissl, J.L. Bosch, The Effect of Dust on Solar Photovoltaic Systems, Energy Procedia, Volume 49, 2014, 2370-2376

[7] A. Sayyah, M. N. Horenstein, M. K. Mazumder, "Energy yield loss caused by dust deposition on photovoltaic panels", Solar Energy, Volume 107, September 2014, Pages 576-604 
[8] Julius Tanesab, David Parlevliet, Jonathan Whale, Tania Urmee, Trevor Pryor, "The contribution of dust to performance degradation of PV modules in a temperate climate zone", Solar Energy, Volume 120, October 2015, $147-157$

[9] Soteris A. Kalogirou, Rafaela Agathokleous, Gregoris Panayiotou, On-site PV characterization and the effect of soiling on their performance, Energy, Volume 51, 1 March 2013, 439-446

[10] S. Silvestre, A. Boronat, A. Chouder, "Study of bypass diodes configuration on PV modules", Applied Energy, Volume 86, Issue 9, September 2009, 1632-1640

[11] Y. Zhao, B. Lehman, R. Ball, J. Mosesian, J. F. de Palma, “Outlier detection rules for fault detection in solar photovoltaic arrays", Applied Power Electronics Conference and Exposition (APEC), 2013 Twenty-Eighth Annual IEEE, 2913 - 2920

[12] T. Takashima, J. Yamaguchi, K. Otani, K. Kato, and M. Ishida, "Experimental studies of failure detection methods in PV module strings”, Photovoltaic Energy Conversion, Conference Record of the 2006 IEEE 4th World Conference, Page(s): 2227-2230

[13] Y. Hirata, S. Noro, T. Aoki, and S. Miyazawa, "Diagnosis Photovoltaic Failure by Simple Function Method to Acquire I-V Curve of Photovoltaic Modules String", Photovoltaic Specialists Conference (PVSC), 2012 38th IEEE, Page(s): 1340-1343

[14] M. Bressan, Y. El-Basri, C. Alonso, "A new method for fault detection and identification of shadows based on electrical signature of defects," in Power Electronics and Applications (EPE'15 ECCE-Europe), 2015 17th European Conference on, vol., no., pp.1-8, 8-10 Sept. 2015

[15] Y. El Basri, M. Bressan, L. Seguier, H. Alawadhi, C. Alonso, “A proposed graphical electrical signatures supervision method to study PV module failures", Solar Energy, Volume 116, June 2015, Pages 247-256

[16] Riley, Cameron William, "An Autonomous Online I-V Tracer for PV Monitoring Applications. " Master's Thesis, University of Tennessee, 2014.

[17] G. Walker "Evaluating MPPT converter topologies using a MATLAB PV model”, J. Electr. Electron. Eng. Aust., vol.21, no. 1, pp 49-56, 2001

[18] M.G. Villalva, J. R. Gazoli, E. R. Filho, “Comprehensive approach to modeling and simulation of photovoltaic arrays”, IEEE transactions on power electronics, Vol. 24, NO. 5, May 2009

[19] J.W Bishop, "Computer Simulation of the effects of electrical mismatches in photovoltaic cell interconnection circuit”, ESTI Project, Commission of the European Communities Joint Research Centre, 1988.

[20] W. Hermann, W. Wiesner, W. Vaaben, "Hot spot investigations on PV modules-new concepts for a test standard and consequences for module design with respect to bypass diodes", Photovoltaic Specialists Conference, 1997., Conference Record of the Twenty-Sixth IEEE, Page(s): 1129-1132

[21] Solórzano, J., Egido, M.A., 2013. “Automatic fault diagnosis in PV systems with distributed MPPT”. Energy Convers. Manage. 76, 925-934.

[22] Tae Hee Jung, Jae Woo Ko, Gi Hwan Kang, Hyung Keun Ahn, "Output characteristics of PV module considering partially reverse biased conditions”, Solar Energy, Volume 92, June 2013, Pages 214-220

[23] Patel H, Agarwal V. "Maximum power point tracking scheme for PV systems operating under partially shaded conditions". IEEE Trans Industr Electron 2008;55(4):1689-98

[24] Bidram A, Davoudi A, Balog RS. "Control and circuit techniques to mitigate partial shading effects in photovoltaic arrays". IEEE J Photovoltaics 2012;2:532-46. 
[25] M. L. Orozco-Gutierrez, J. M. Ramirez-Scarpetta, G. Spagnuolo, and C. A. Ramos-Paja, "A method for simulating large PV arrays that include reverse biased cells," Applied Energy, vol. 123, pp. 157-167, Jun. 2014. [26] F.Martínez-Moreno, J. Muñoz, E. Lorenzo, "Experimental model to estimate shading losses on PV arrays", Solar Energy Materials \& Solar Cells 94 (2010) 2298-2303

[27] M. Drif, A. Mellit, J. Aguilera, P. J. Pérez, “A comprehensive method for estimating energy losses due to shading of GC-BIPV systems using monitoring data”, Solar Energy 86 (2012) 2397-2404

[28] L. Sun, L. Lu, H. Yang, "Optimum design of shading-type building-integrated photovoltaic claddings with different surface azimuth angles", Applied Energy 90 (2012) 233-240 [29] M.C. Alonso-García, J.M. Ruiz, W. Herrmann, "Computer simulation of shading effects in photovoltaic arrays", Renewable Energy, Volume 31, Issue 12, October 2006, Pages 1986-1993 [30] Engin Karatepe, Mutlu Boztepe, Metin Çolak, "Development of a suitable model for characterizing photovoltaic arrays with shaded solar cells”, Solar Energy, Volume 81, Issue 8, August 2007, Pages 977-992, [31] Hajime Kawamura, Kazuhito Naka, Norihiro Yonekura, Sanshiro Yamanaka, Hideaki Kawamura, Hideyuki Ohno, Katsuhiko Naito, "Simulation of I-V characteristics of a PV module with shaded PV cells", Solar Energy 